\title{
Tunnel injection $\ln _{0.4} \mathrm{Ga}_{0.6} \mathrm{As} / \mathrm{GaAs}$ quantum dot lasers with $15 \mathrm{GHz}$ modulation bandwidth at room temperature
}

\author{
P. Bhattacharya ${ }^{\text {a) }}$ and S. Ghosh \\ Solid State Electronics Laboratory Department of Electrical Engineering and Computer Science \\ University of Michigan, Ann Arbor, Michigan 48109-2122
}

(Received 2 January 2002; accepted for publication 13 March 2002)

\begin{abstract}
By utilizing tunnel injection of electrons, first demonstrated in quantum well lasers, we have measured enhanced small-signal modulation bandwidth, $f_{-3 \mathrm{~dB}}$, and reduced temperature sensitivity of the threshold current, characterized by $T_{0}$, in $\operatorname{In}_{0.4} \mathrm{Ga}_{0.6} \mathrm{As} / \mathrm{GaAs}$ self-organized quantum dot ridge waveguide lasers. Values of $f_{-3 \mathrm{~dB}}=15 \mathrm{GHz}$ at $283 \mathrm{~K}$ and $T_{0}=237 \mathrm{~K}$ for $318 \geqslant T \geqslant 278$ are measured in these devices. The differential gain at $283 \mathrm{~K}$ is $d g / d n \cong 8.5 \times 10^{-14} \mathrm{~cm}^{2}$ and the gain compression factor $\epsilon=4.5 \times 10^{-17} \mathrm{~cm}^{3}$. (c) 2002 American Institute of Physics.
\end{abstract}

[DOI: $10.1063 / 1.1478129$ ]

Very promising performance characteristics of $\operatorname{In}(\mathrm{Ga}) \mathrm{As} /$ GaAs quantum dot (QD) lasers have been reported in the recent past in terms of threshold current, tunability of wavelength, and output power. ${ }^{1-3}$ Values of differential gain as high as $10^{-13} \mathrm{~cm}^{2}$ at $77 \mathrm{~K}^{4}$ have been measured under high injection conditions in QD lasers. In spite of this, the measured modulation bandwidth of $1 \mu \mathrm{m}$ and $1.3 \mu \mathrm{m}$ singlemode high-speed QD lasers is typically limited to 5-6 GHz. ${ }^{5-7}$ From analysis of high frequency electrical impedance measurement data of single-mode QD lasers, with the appropriate carrier and photon rate equations, a "quantum capture" time of injected carriers is derived. ${ }^{8}$ The value of this parameter, which includes transport through the heterostructure, continuum to bound capture time and intersubband carrier relaxation, varies with injection current and number of dot levels in the active region. For example, in a $\mathrm{In}_{0.4} \mathrm{Ga}_{0.6} \mathrm{As} / \mathrm{GaAs}$ multidot layer laser the quantum capture time is approximately $30-50 \mathrm{ps}$, which agrees with the results of Heitz et al., ${ }^{9}$ and also quantitatively agrees with the measured modulation bandwidth of $5 \mathrm{GHz}$. More direct femtosecond differential transmission measurements have been made by us on similar QD heterostructures using the pumpprobe technique. ${ }^{5}$ These measurements have been done as a function of temperature $(4-300 \mathrm{~K})$ and excitation level (number of carriers per dot). From a careful analysis of the data, it is apparent that in addition to intersubband electron relaxation in the dots, carrier re-emission to the barrier and wetting layers and the density of available states in these regions also play significant roles in determining the carrier dynamics, particularly at elevated temperatures. Similar conclusions were also arrived at by Deppe et al. ${ }^{10}$ Depending on excitation level and temperature, intersubband relaxation times vary from a few ps to $\sim 100$ ps. ${ }^{11}$ It is useful to note that in quantum wells, the relaxation times are $\sim 2-5$ ps. ${ }^{12}$

It is evident that a significant "hot-carrier" problem, or a gain compression can exist in QD lasers at the wavelength of the ground state transition. It is therefore worthwhile to explore alternate techniques of injecting electrons directly to the lasing states, thereby bypassing the usual injection pro-

${ }^{a)}$ Electronic mail: pkb@eecs.umich.edu cess over the heterojunction barriers. It has been demonstrated by us that tunneling of electrons directly into the lasing subband improves the performance characteristics of quantum-well lasers. ${ }^{13}$ Higher small-signal modulation bandwidth, lower wavelength chirp, reduced Auger recombination, and a smaller temperature dependence of the threshold current (large $T_{0}$ ), compared to conventional separate confinement heterostructure $(\mathrm{SCH})$ lasers, were measured in GaAs- and InP-based tunnel injection lasers. ${ }^{13-15}$ By tunnel injection, "cold" electrons are introduced into the lasing subband of the active (gain) region (resonantly or phonon assisted) at or near the Fermi level at a rate higher than the stimulated emission rate, and the electron distribution remains quasi-Fermi even at large drive currents. The hole thermalization rates are usually very large due to the high density of states and band mixing. Hot-carrier effects, mentioned earlier, can therefore be minimized. Asyran and Lury ${ }^{16}$ have recently shown from theoretical considerations that using tunnel injection, parasitic recombination of carriers outside the quantum dot can be greatly reduced in a QD laser, and have predicted large values of the parameter $T_{0}$. The tunneling concept has also been recently demonstrated in photopumped InP QD lasers. ${ }^{17}$ In this letter we demonstrate large room-temperature modulation bandwidth $(\sim 15$ $\mathrm{GHz}$ ) and large $T_{0}$ in self-organized $\mathrm{In}_{0.4} \mathrm{Ga}_{0.6} \mathrm{As} / \mathrm{GaAs} \mathrm{QD}$ lasers wherein electrons are injected into the quantum dots by phonon assisted tunneling.

The QD laser heterostructure grown by molecular beam epitaxy is shown in the inset of Fig. 1(a) and the conduction band profile is illustrated in Fig. 1(b). Transmission electron microscopy of the heterostructures does not reveal any dislocations. Low-temperature photoluminescence (PL) measurements were performed on the samples with a $0.75 \mathrm{~m}$ scanning spectrometer, liquid nitrogen cooled photomultiplier, and lock-in amplification. The samples exhibit luminescence peaks at 1.18 and $1.27 \mathrm{eV}$ from the QDs and from the $\mathrm{In}_{0.23} \mathrm{Ga}_{0.77} \mathrm{As}$ quantum well injector, respectively. Taking the hole confinement energies into account, the electronic energy separation in the conduction band, which is relevant to the tunneling process, is $\Delta E=82 \mathrm{meV}$. Rapid thermal annealing of quantum dots is known to blue-shift the ground 


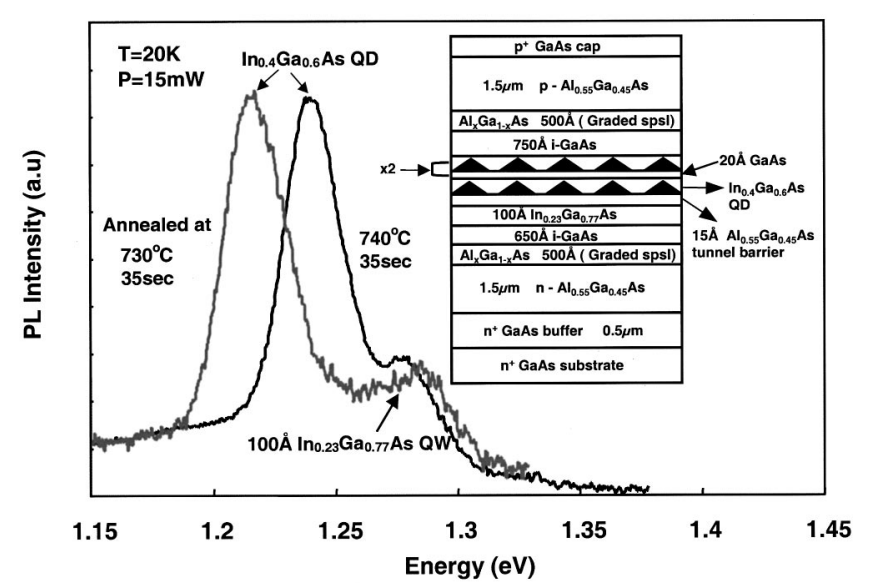

(a)

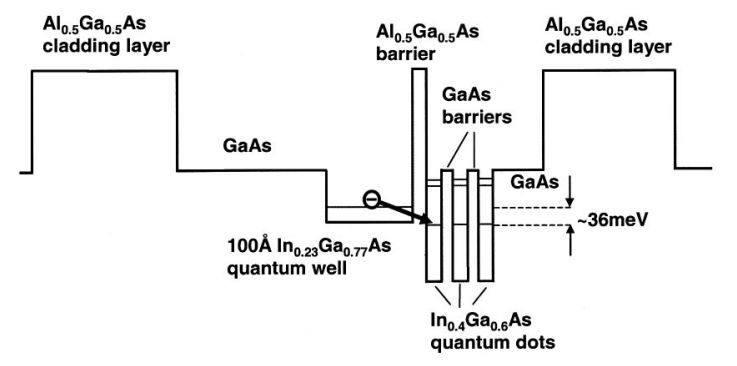

(b)

FIG. 1. (a) The low temperature photoluminescence spectra of the rapid thermal annealed laser heterostructure under different annealing conditions. The inset shows the schematic of tunnel injection quantum dot laser heterostructure grown by molecular beam epitaxy; (b) the flat-band profile of the conduction band across the heterostructure.

and excited state transition energies due to In-Ga interdiffusion at the $\mathrm{In}_{0.4} \mathrm{Ga}_{0.6} \mathrm{As} / \mathrm{GaAs}$ boundary, and partly to a dot size reduction. ${ }^{18}$ The shift in the quantum well peak is very small under similar annealing conditions. Our objective was to reduce the energy difference $\Delta E$ between the injector well and the ground state of the quantum dot to $\sim 36 \mathrm{meV}$, such that electrons can be injected from the quantum well to the dot ground state, and not the excited state, by phonon assisted tunneling. This was achieved by annealing the heterostructure at $740{ }^{\circ} \mathrm{C}$ for $35 \mathrm{~s}$. The measured $\Delta E$ varies in the range of $25-30 \mathrm{meV}$ at $20 \mathrm{~K}$ as shown in Fig. 1(a). Temperature dependent PL measurements on the laser heterostructure show that the $\Delta E$ increases with temperature at a rate of $\sim 5.5 \times 10^{-2} \mathrm{meV} / \mathrm{K}$. Therefore, the $\Delta E$ is $\sim 36 \mathrm{meV}$ at room temperature.

Ridge waveguide lasers $5 \mu \mathrm{m} \times 400 \mu \mathrm{m}$ long were made by standard photolithography, wet and dry etching, and metallization techniques. The threshold current of the laser at $300 \mathrm{~K}$ was measured to be $40 \mathrm{~mA}$, which is rather high. We believe this is due to increased waveguide losses (measured to be $9.8 \mathrm{~cm}^{-1}$ ) in the tunneling structure, the small fill factor of the dots $(\sim 0.28)$ and the relatively small cavity length (very small values of $I_{\mathrm{th}}$ have been reported in laser $\sim 1 \mathrm{~mm}$ long). The lasing peak at threshold is at $1022 \mathrm{~nm}(1.21 \mathrm{eV})$. The small-signal modulation response of the laser was measured at room temperature with a HP $8562 \mathrm{~A}$ electrical spectrum analyzer, a HP 8350B sweep oscillator, a low noise amplifier, and a New Focus high-speed detector. The frequency response for varying injection currents is shown in

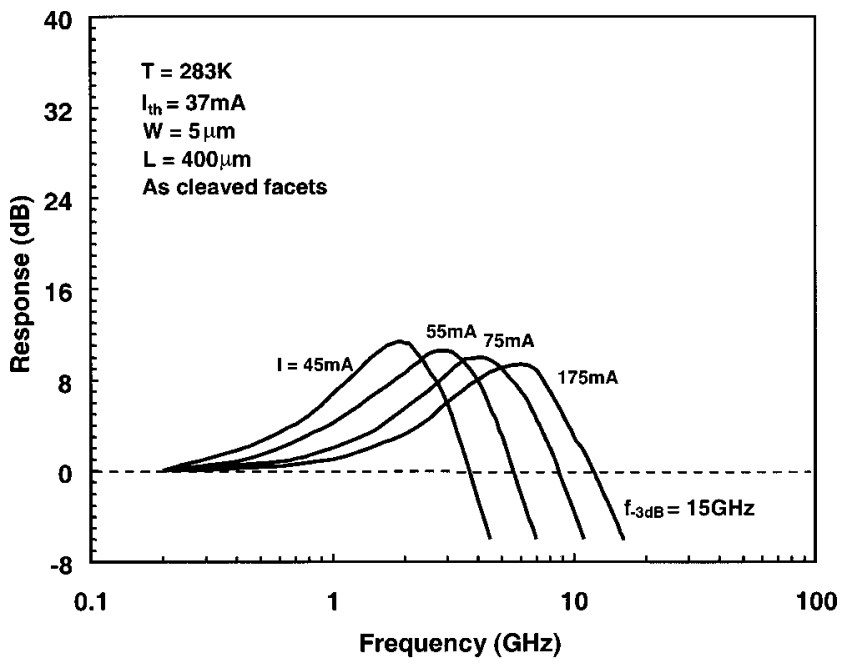

(a)

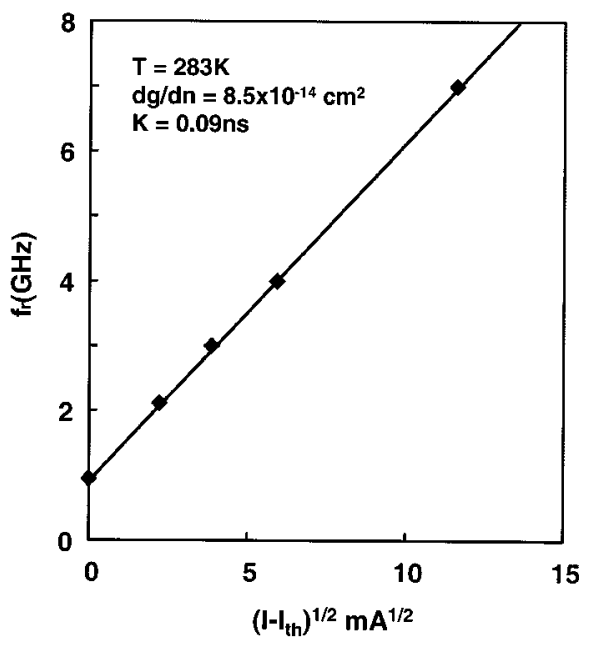

(b)

FIG. 2. (a) Measured small-signal modulation response of tunnel injection QD laser at $283 \mathrm{~K}$ for varying injection currents; (b) plot of the resonance frequency of the modulation response versus the square root of $\left(I-I_{\mathrm{th}}\right)$.

Fig. 2(a). A bandwidth of $f_{-3 \mathrm{~dB}}=15 \mathrm{GHz}$ is measured for $I$ $=175 \mathrm{~mA}$ under pulsed operation $(1 \mu \mathrm{s}$ pulses at $10 \mathrm{KHz})$. The devices were also tested under continuous wave operation and a bandwidth of $\sim 12 \mathrm{GHz}$ was measured for $I$ $=175 \mathrm{~mA}$. Figure 2(b) shows a plot of the resonance frequency $f_{r}$ of the modulation response, under pulsed condition, versus the square root of the injection current, which has a slope of $0.53 \mathrm{GHz} / \mathrm{mA}^{1 / 2}$. With this value of the slope, a value of $28 \%$ for the QD fill factor as measured by atomic force microscopy on similar samples, and a confinement factor of $\Gamma=2.4 \times 10^{-3}$ for the laser heterostructure, ${ }^{5}$ a value of $d g / d n=8.5 \times 10^{-14} \mathrm{~cm}^{2}$ is obtained. This value of differential gain is considerably higher than that measured in conventional SCH QD lasers ${ }^{5}$ grown under identical conditions.

The measured small-signal modulation bandwidth of 15 $\mathrm{GHz}$, which is the highest to our knowledge at room temperature, indicates that QD lasers can exhibit high-speed modulation bandwidths once carriers are injected at the lasing energy in the dot, and that most of the recombination takes place therein. The presence of the $100 \AA \mathrm{In}_{0.23} \mathrm{Ga}_{0.77} \mathrm{As}$ injector QD helps to inject electrons and keep them localized in the dots. It is also important to note that a bandwidth of 15 


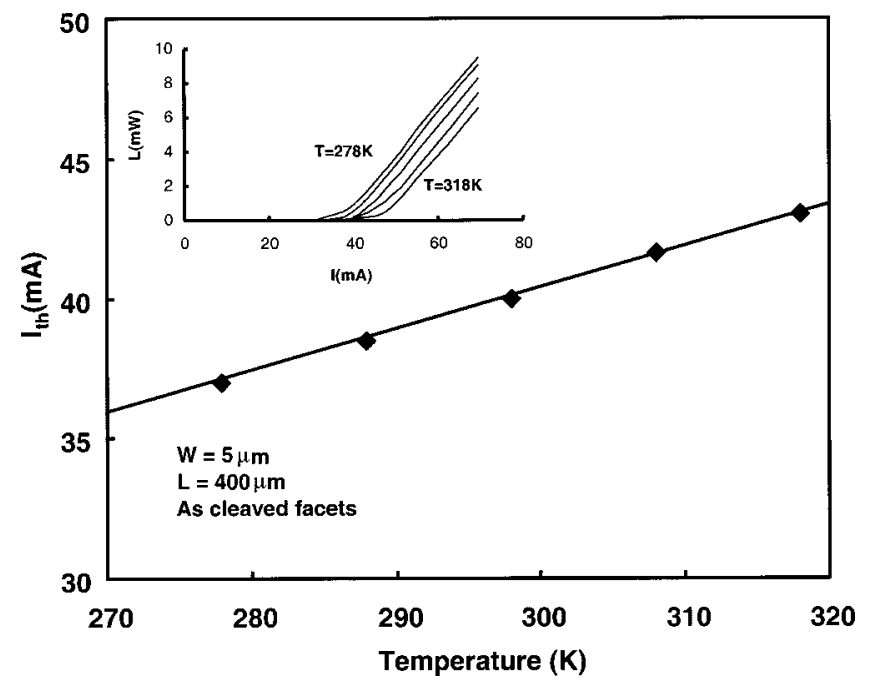

FIG. 3. Variation of measured $I_{\mathrm{th}}$ with temperature. The line is the fit to the data in accordance with $I_{\mathrm{th}}(T)=I_{\mathrm{th}}(0) e^{T / T_{0}}$. Shown in the inset are the measured light-current characteristics in QD tunnel injection laser.

$\mathrm{GHz}$ is measured in a laser which is $400 \mu \mathrm{m}$ long and with a injection current ratio $I / I_{\text {th }}$ as low as 4.5. To get higher modulation bandwidths, it is necessary to increase the photon density in the cavity, which is attained with a high injection current and a very short length $(\sim 100 \mu \mathrm{m})$ cavity. Preliminary calculations of the small-signal modulation response with the material parameters of $\mathrm{In}_{0.4} \mathrm{Ga}_{06}{ }_{6}$ As QDs, assuming that the strain in these dots is largely biaxial due to their shape, indicate that $f_{-3 \mathrm{~dB}}$ should be $30-35 \mathrm{GHz}$ in welldesigned devices. ${ }^{13}$ It should be noted that the high frequency modulation response characteristics display a dominant peak at $\omega=\omega_{r}$, the resonance frequency. This feature is characteristic of all tunnel injection lasers and, to a large extent, indicates that gain compression effects are minimized in these devices. Analysis of the modulation response yields a gain compression factor $\epsilon=4.5 \times 10^{-17} \mathrm{~cm}^{3}$. This value is a factor of 100 lower than SCH QD lasers.

Figure 3 shows the measured variation of $I_{\text {th }}$ with $T$. A value of $T_{0}=237 \mathrm{~K}$ is obtained from a fit to the data in accordance with $I_{\mathrm{th}}(T)=I_{\mathrm{th}}(0) e^{T / T_{\mathrm{o}}}$. The slope efficiency of $29 \%$ indicates that nonradiative recombination could contribute to the large value of $T_{0}$. However, comparing our measured values of $T_{0}$ with calculated data of Asyran and Lyuri, ${ }^{16}$ it appears that fraction of injected carriers that recombines in the dots is quite high. This is to be expected since carrier loss from the dots and recombination in other regions of the heterostructure are minimized by the tunnel injection scheme.

In conclusion, by phonon assisted tunnel injection of electrons in a $\operatorname{In}_{0.4} \mathrm{Ga}_{0.6} \mathrm{As} / \mathrm{GaAs}$ QD laser, we demonstrate a large modulation bandwidth of $15 \mathrm{GHz}$ at room temperature. The tunnel injection process helps to maintain a quasi-Fermi distribution in the quantum dot, reduces gain compression and minimizes recombination of carriers in regions outside the quantum dot.

The work is supported by the Air Force Office of Scientific Research (MURI Program) under Grant No. F49620-001-0328 and the Army Research Office under Grant No. DAAD 19-01-1-0331. One of the authors (P.B.) thanks General Dynamics Corporation for the support of the James R. Mellor Chair.

${ }^{1}$ G. T. Liu, A. Stintz, H. Li, K. J. Malloy, and L. F. Lester, Electron. Lett. 35, 1163 (1999).

${ }^{2}$ P. M. Varangis, H. Li, G. T. Liu, T. C. Newell, A. Stintz, B. Fuchs, K. J. Malloy, and L. F. Lester, Electron. Lett. 36, 1544 (2000).

${ }^{3}$ R. L. Sellin, Ch. Ribbat, M. Grundmann, N. N. Ledenstov, and D. Bimberg, Appl. Phys. Lett. 78, 1207 (2001).

${ }^{4}$ N. Kirstaedter, O. Schmidt, N. Ledenstov, D. Bimberg, V. Ustinov, A. Egorov, A. Zhukov, M. Maximov, P. Kop'ev, and Zh. I. Alferov, Appl. Phys. Lett. 69, 1226 (1996).

${ }^{5}$ P. Bhattacharya, K. K. Kamath, J. Singh, D. Klotzkin, J. Phillips, H-T. Jiang, N. Chervela, T. Norris, T. Sosnowski, J. Laskar, and M. R. Murty, IEEE Trans. Electron Devices 46, 871 (1999).

${ }^{6}$ D. Klotzkin, K. Kamath, K. Vineberg, P. Bhattacharya, R. Murty, J. Laskar, IEEE Photonics Technol. Lett. 10, 932 (1998).

${ }^{7}$ R. Krebs, F. Klopf, S. Rennon, J. P. Reithmaier, and A. Forchel, Electron. Lett. 37, 1223 (2001).

${ }^{8}$ D. Klotzkin, K. Kamath, and P. Bhattacharya, IEEE Photonics Technol. Lett. 9, 1301 (1997)

${ }^{9}$ R. Heitz, M. Veit, N. N. Ledenstov, A. Hoffman, D. Bimberg, V. M. Ustinov, P. S. Kop'ev, and Zh. I. Alferov, Phys. Rev. B 56, 10435 (1997).

${ }^{10}$ D. G. Deppe and D. L. Huffaker, Appl. Phys. Lett. 77, 3325 (2000).

${ }^{11}$ S. Krishna, P. Bhattacharya, J. Urayama, J. Singh, T. Norris, P. J. McCann, and K. Namjou, IEEE J. Quantum Electron. 37, 1066 (2001).

${ }^{12}$ L. Davis, Y. L. Lam, Y. C. Chen, J. Singh, and P. Bhattacharya, IEEE J. Quantum Electron. 30, 2560 (1994).

${ }^{13}$ P. Bhattacharya, J. Singh, H. Yoon, X. Zhang, A. Gutierrez-Aitken, and Y. Lam, IEEE J. Quantum Electron. 32, 1620 (1996).

${ }^{14}$ H. Yoon, A. Gutierrez-Aitken, R. Jambunathan, J. Singh, and P. Bhattacharya, IEEE Photonics Technol. Lett. 7, 974 (1992).

${ }^{15}$ H. Yoon, H. C. Sun, and P. Bhattacharya, Electron. Lett. 30, 1675 (1994).

${ }^{16}$ L. Asryan and S. Luryi, IEEE J. Quantum Electron. 37, 905 (2001).

${ }^{17}$ G. Walter, N. Holonyak, Jr., J. H. Ryou, R. D. Dupuis, Appl. Phys. Lett. 79, 1956 (2001)

${ }^{18}$ S. J. Xu, X. C. Wang, S. J. Chua, C. H. Wang, W. J. Fan, J. Jiang, and X. G. Xie, Appl. Phys. Lett. 72, 3335 (1998). 